\title{
REVIEW
}

\section{Zinc fingers and homeoboxes family in human diseases}

\author{
Y Liu, D Ma and C Ji
}

The zinc-fingers and homeoboxes (ZHX) family is a group of nuclear homodimeric transcriptional repressors that interact with a subunit of nuclear factor-Y (NF-YA) and contain two $\mathrm{C} 2 \mathrm{H} 2$-type zinc fingers and five homeobox DNA-binding domains. The members of ZHX family form homodimers or heterodimers with other members or a subunit of NF-YA to repress transcription. ZHX family members function in hematopoietic cell development and differentiation, and neural progenitor maintenance. Dysfunction of ZHX family members correlates with the development and progression of various diseases, including hepatocellular carcinoma (HCC), hematological diseases, neurological diseases and glomerular diseases. Furthermore, low expression of ZHX is associated with poor prognosis in malignancies. This review provides an update on the role of ZHX family in development and its function in cancer, with special emphasis on HCC and hematological malignant diseases.

Cancer Gene Therapy (2015) 22, 223-226; doi:10.1038/cgt.2015.16; published online 10 April 2015

\section{THE ZHX FAMILY}

The zinc-fingers and homeoboxes (ZHX) family includes ZHX1, ZHX2, and ZHX3.$^{1-3}$ All ZHX family members contain two Cys-Xaa2-Cys-Xaa12-His-Xaa4-His-type zinc-finger motifs and four or five HOX-like homeodomains (HDs), and function as transcriptional repressors (Figure 1a). ${ }^{3,4}$ The type and component of homeodomains in the $\mathrm{ZHX}$ family are restricted to vertebrate lineage. The human $\mathrm{ZHX1}$ and $\mathrm{ZHX} 2$ are on chromosome 8, whereas ZHX3 is on chromosome 20.

ZHX1 was firstly identified in a bone marrow stromal cell complementary DNA (cDNA) library by yeast two-hybrid library screen in 1999. ${ }^{1,5}$ The 873 amino acid ZHX1 protein contains two $\mathrm{N}$-terminal zinc fingers, five central and C-terminal homeodomains, a C-terminal acidic region, and two putative nuclear localization signals (NLSs). The expression of ZHX1 is high in brain and low in liver and kidney, whereas nearly undetectable in heart and muscle. ${ }^{1,5,6}$ The human and mouse ZHX1 proteins share $91 \%$ amino acid identity.

Human ZHX2 was cloned from a size-fractionated brain cDNA library in 1998. It contains two C2H2-type zinc-finger motifs and five $\mathrm{HDs}$, with a unique proline-rich region ( $\mathrm{P}$ domain) between HD1 and HD2 (Figure 1a). ${ }^{3}$ ZHX2 expression was detected in all kinds of tissues with highest levels in ovary, followed by lung, heart, kidney, brain and liver. Intermediate expression was detected in pancreas, spleen, testis and skeletal muscle. ${ }^{7}$ Mouse and rat ZHX2 were cloned in 2003 by database analysis, respectively. $^{2}$ The mouse and human ZHX2 proteins share $87 \%$ amino acid identity. Besides, some $\mathrm{ZHX} 2$ variants have been identified. G779A polymorphism of ZHX2 has been identified (Figure 1b). ${ }^{8}$ However, further function studies are required for this polymorphism. A polymorphism of ZHX2 in intron 2 has also been identified. Individuals with this polymorphism have been demonstrated with the strong response to smallpox vaccine in a genome-wide association study. ${ }^{9}$

A partial human ZHX3 CDNA, KIAA0395, was identified in 1997. ${ }^{10}$ Three full-length human ZHX3 CDNA were subsequently identified in a testis cDNA library. ZHX3 protein was eventually cloned by screening rat liver and granulose cell cDNA libraries using yeast two-hybrid analysis with ZHX1 as bait in $2003 .{ }^{4} \mathrm{ZHX} 3$ encodes a 956 amino acid protein with two zinc-finger domains, five homeodomains, a glu-rich region (E domain), and two NLS (Figure 1a). ZHX3 and ZHX1 share $34.4 \%$ amino acid identity.

\section{ZHXS SIGNALING}

ZHX family molecules are involved in the development and differentiation in different types of cells, and always act as transcriptional repressors by binding with the promoter regions to regulate the transcription of target genes in human tissues. Current studies demonstrated that a direct interaction between ZHX1 and ZHX2 could not only form heterodimer, but also form homodimers in vivo and in vitro to repress transcription. ${ }^{2,3,11}$ All interactions required an extensive region around HD1. ZHX proteins also interact with nuclear factor-Y (NF-Y) subunits to have transcriptional suppression roles. NF-Y family contains three subunits NF-YA, NF-YB and NF-YC. NF-YA subunit includes two activation domains, a serine/threonine-rich region and a glutamine-rich region. ZHX proteins interact with different activation domains of NF-YA. Another transcriptional co-repressor of ZHX1, BS69, has also been identified. ${ }^{12}$ ZHX1 interacts with the glutamine-rich region, whereas $\mathrm{ZHX} 2$ and $\mathrm{ZHX} 3$ interact with the serine/threoonine-rich region.,13 Meanwhile, the NLS of ZHX proteins are different. The NLS of ZHX1 is located in the amino acid sequence between residues 734 and $768,{ }^{14}$ which contains an arginine-rich basic region. The NLS of ZHX2 is sited at a prolinerich basic region, corresponding to the amino acid sequence between residues 317 and 446 (ref. 3; Figure 1a). In contrast, ZHX3 contains two NLSs that are located in the N-terminal zinc-finger 1 and HD2 region. ${ }^{4}$ The repressor domain of ZHX1 is the C-terminal acidic region, which corresponds to the amino acid sequence between residues 831 and $873 .^{14}$ Both ZHX2 and ZHX3 contain a transcriptional repressor domain in the HD1, corresponding to the 
amino acid sequence between residues 263 and 446, and between residues 303 and 502, respectively. ${ }^{3,4}$ The dimerization with ZHX1, ZHX2 or ZHX3 is requested for full repressor activities. $^{4,14}$

The upstream regulators and downstream targets of ZHX2 have been demonstrated. As shown in Figure 2a, FoxC1 and ephrin-B1/B2 are identified as upstream regulators of ZHX2. Specifically, FoxC1 represses the expression of $Z \mathrm{HX} 2$, whereas ephrin-B1/B2 activates the ZHX2 signaling pathways. ${ }^{15,16}$ In the downstream signaling of ZHX2, ZHX2 represses the expression of AFP, Cyclin A and Cyclin E. In vitro, cellular studies also demonstrated that ZHX2 induces the activity of lipoprotein lipase (Lpl). ${ }^{17}$ However, most of the studies about ZHX2 signaling are very preliminary. Further studies are required to demonstrate more details about $\mathrm{ZHX} 2$ signaling.

It has been shown that ZHX family can regulate alphafetoprotein (AFP) expression in liver development. The expression of ZHX2 protein is absent in the fetal liver but upregulated in the normal adult liver. However, the expression of ZHX2 in hepatocellular carcinoma (HCC) exhibits an opposite tendency, which is dramatically repressed. ${ }^{18,19}$ This tendency is different with the expression of AFP protein in liver development and diseases. ${ }^{20}$ Studies in mice also demonstrated that $\mathrm{ZHX} 2$ is a negative transcriptional regulator of AFP in liver. $^{18,21}$ The molecular mechanism that ZHX2 repressed AFP expression has also been demonstrated. Specifically, AFP regulator 1 is an important regulatory factor of AFP and targets to AFP promoter to repress the transcription of AFP. Furthermore, Perincheri et al. ${ }^{18}$ used the positional cloning approach to demonstrate that AFP regulator 1 protein binds with ZHX2 protein to forma complex. They also found that AFP expression in BALB/cJ mouse liver was caused by the ZHX2 mutation. Shen et al. ${ }^{22}$ found ZHX2 interacted with

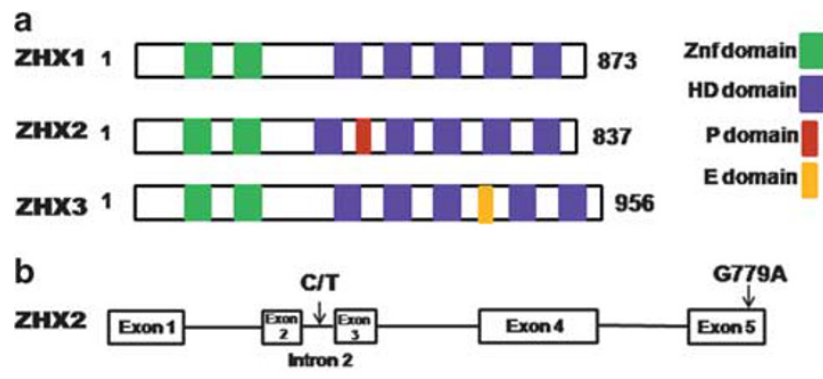

Figure 1. Diagram of zinc fingers and homeoboxes (ZHX) family. (a) Protein structures of ZHX family. (b) Gene structure of the polymorphisms of $Z H X 2$. hepatocyte NF-1 binding sites to repress AFP transcription in HCC cell lines. Furthermore, a clinical study demonstrated that ZHX2 expression is negatively related with AFP expression in HCC samples. ${ }^{23}$ Other genes, such as Gpc3 and H19 were also demonstrated to regulate AFP expression controlled by ZHX2 ${ }^{18,24}$ These results were further confirmed by partial hepatectomy in mouse. The level of ZHX2 was repressed at 24 and $48 \mathrm{~h}$ after partial hepatectomy, whereas the levels of AFP and GPC3 increased significantly. Similar to AFP and GPC3, the ZHX2 expression recovered to normal level after $48 \mathrm{~h}^{25}$

\section{ZHX FAMILY MEMBERS IN HUMAN DISEASES}

Increasing evidence confirms important roles of ZHX family in the development of human diseases. Several studies indicated that the low expression of ZHX was bound up with the process of tumorigenesis and development. As a tumor suppressor gene, ZHX2 is of great significance in the diagnosis of tumor diseases (Figure $2 \mathrm{~b}$ ).

\section{HEPATOCELLULAR CARCINOMA}

Several independent groups have demonstrated that overexpression of ZHX2 inhibited the growth of HCC cells in vivo and in vitro. ${ }^{18,21}$ Furthermore, high level of $\mathrm{ZHX2}$ expression was correlated with low expression of Cyclin A and Cyclin E in HCC samples, suggesting that ZHX2 induces cell cycle arrest at G1 phase. ${ }^{21}$ Mechanistic studies indicated that ZHX2 protein directly bound with the promoter regions of Cyclin A and Cyclin E to repress their transcriptions. ${ }^{21}$

Repression of ZHX2 in HCC has also been identified. Lv et al. ${ }^{26}$ reported that hypermethylation of the ZHX2 promoter was higher in HCC than in adjacent non-tumor issues using methylationsensitive restriction fingerprinting. They also found that the expression of ZHX2 was silenced in liver of HCC patients. These results suggest that $\mathrm{ZHX} 2$ is a tumor suppressor. Wang et al. ${ }^{27}$ have confirmed the similar results independently. However, $\mathrm{Hu}$ et $\mathrm{al}^{28}$ demonstrated that the expression of ZHX2 was upregulated in HCC samples comparing with the adjacent nontumor issues, especially in poorly differentiated and metastasis samples. It's a controversial problem that needs to be explored.

The clinical significance of ZHX2 expression in HCC has been demonstrated. The overexpression of $\mathrm{ZHX} 2$ was detected in well-differentiated liver tissues than in poor-differentiated tissues. ${ }^{21}$ Furthermore, nuclear ZHX2 expression was also correlated with the overall survival times of patients and was correlated with the inhibition of hepatocyte proliferation and tumor microvascularization. ${ }^{21}$
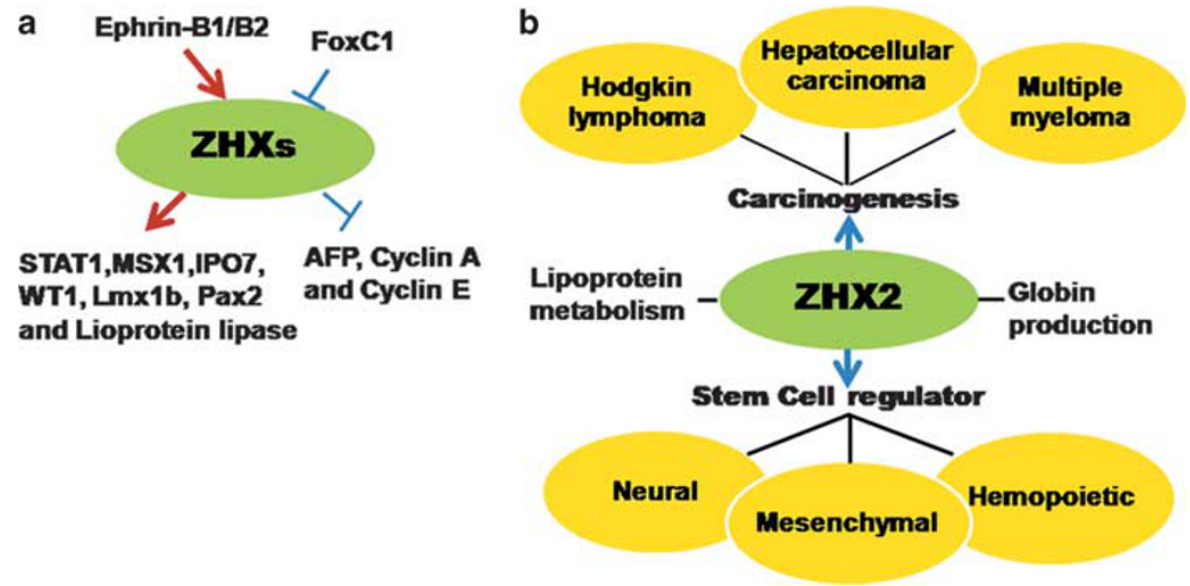

Figure 2. ZHX2 in different kinds of human disease. (a) ZHX2 signaling. (b) ZHX2-related diseases. 


\section{HEMATOLOGICAL DISEASES}

ZHX2 gene coincides on the quantitative trait loci that have been reported to influence the absolute fetal hemoglobin levels. ${ }^{29}$ de Andrade et al. $^{30}$ demonstrated that the expressed transcripts of ZHX2 in reticulocytes from a normal and a hereditary persistence of fetal hemoglobin-2 patients were different. Some of the detectable transcripts were involved in globin gene regulation. ${ }^{30}$ Downregulation of $\mathrm{ZHX} 2$ was detected in two hereditary persistence of fetal hemoglobin-2 patients and in a carrier of the Sicilian $\delta \beta$-thalassemia trait. These results suggest that ZHX2 represses the expression of globin, particularly $\gamma$-globin. ${ }^{30}$ The clinical manifestations of $\beta$-thalassemia are extremely variable in severity. It has been reported that there is no apparent relationship among G779A polymorphism of ZHX2 gene and severity of thalassemia and the level of HbF8. It has also been found that ZHX2 protein was involved in red blood cell differentiation and its expression was influenced by erythropoietin. ${ }^{31}$

Recent study also confirmed that ZHX2 was aberrantly expressed in multiple myeloma (MM). ${ }^{32}$ The critical role of ZHX2 in $M M$ was initially identified in studies of gene-expression profiles. Shaughnessy et al. ${ }^{33}$ found that low expression of ZHX2 was associated with a risk of progression in a selected series of 221 transplanted patients with MM. In addition, lower expression of ZHX2 protein was detected in high-risk proliferative MM than in low-risk proliferative $\mathrm{MM}^{34}$ and the expression level of $\mathrm{ZHX} 2$ protein was closely related with the invasion ability of MM. ${ }^{35}$ Some properties of ZHX2 are taken into an important prognostic marker for MM. The increased ZHX2 mRNA level was associated with beneficial prognostic index like $\beta_{2}$-microglobulin $<5.5 \mathrm{mgl}^{-1}$, albumin $>35 \mathrm{gl}^{-1}$ or a better cytogenetics. ${ }^{34}$ Furthermore, high expression level of ZHX2 correlated with prolonged duration of response and overall survival of patients. ${ }^{34}$ In addition, the patients with low ZHX2 levels had a higher rate of resistance to chemotherapy. $^{34}$

Expression analysis in hematopoietic cell lines and primary cells indicated that B-cell-specific homeobox gene $Z H X 2$ is a tumor suppressor gene in Hodgkin lymphoma. $t(4 ; 8)(q 27 ; q 24)$ is a novel chromosomal rearrangement in Hodgkin lymphoma. ${ }^{36}$ Target genes at $4 q 27$ or $8 q 24$ were shortlisted. Expression analysis of candidate target genes revealed that the inhibition of homeobox gene ZHX2 was located at $8 \mathrm{q} 24 .^{36} \mathrm{ZHX2}$ is a critical factor in development and differentiation of early $B$ cells. The expression of ZHX2 was upregulated during the process from hematopoietic stem cells to early $B$ stage or from early $B$ to pro-B transitions. ${ }^{37}$ The repression of $\mathrm{ZHX} 2$ is caused by the upregulation of FoxC1. ${ }^{38}$ Aberrantly expressed FoxC1 leads to activation of IPO7 and repression of the transcription factors $M S X 1$, and subsequently repressed the activation region of $Z H X 2$ gene. ${ }^{15}$ IPO7 also contributes to ZHX2 repression by increasing nuclear levels of co-repressor histone $\mathrm{H}_{1} \mathrm{C}^{38}$

\section{OTHERS}

ZHX2 functions in lipoprotein metabolism in vivo. Gargalovic et al. ${ }^{17}$ demonstrated that mutation of $\mathrm{ZHX} 2$ regulates the lipoprotein alterations, especially the expression of $\mathrm{Lpl}$ in the mouse models. Lpl is a critical enzyme in the metabolism of triglyceride-rich lipoproteins. ${ }^{39}$ Some ZHX2 variants activate the expression of Lpl. ${ }^{17}$ However, it remains unclear how ZHX2 protein regulates $\mathrm{Lpl}$ expression. There is no evidence to indicate that ZHX2 binds with Lpl promoter directly. It's possible that ZHX2 forms heterodimers with other family members, such as ZHX3, to indirectly interact with the promoter regions of $\mathrm{Lpl} .{ }^{40}$ Moreover, a mass of ZHX3 mutations were found in both hypertriglyceridemia patients and healthy people. ${ }^{41}$ Thus, the role of different ZHX proteins in lipoprotein metabolism should be further investigated.
ZHX2 protein was specifically detected in the ventricular and subventricular zone of the cortex during various stages of cortical neurogenesis. Blocking ZHX2 signaling in cortical neural progenitor cells by the expression of ZHX2-VP16 causes neuronal differentiation, whereas overexpression of ZHX2 in the cortex interrupts the normal differentiation of cortical neural progenitor cells. ${ }^{42}$ Overexpression of ZHX2 was identified in neural progenitor cell and correlated with the expression of nestin protein. ${ }^{42} \mathrm{ZHX} 2$ is also a candidate NF for the intracellular fragment of ephrin-B, a critical factor in regulating cortical neural progenitors. ${ }^{16,43,44}$ Introduction of an ephrin-B1 intracellular domain can activate ZHX2 activity. ${ }^{42}$ Theephrin-B1 binding domain of ZHX2 is at the $\mathrm{N}$-terminal portion (263-294) of the ephrin-B1 cytoplasmic domain, a region adjacent to the transmembrane domain and conserved between ephrin-B1 and ephrin-B2. ${ }^{42}$ In addition, ZHX2 variants have also been identified in two corticobasal degeneration patients. These results further indicate that $\mathrm{ZHX} 2$ participates in the genesis and development of human neurological disease. ${ }^{45}$

Recent studies suggest that ZHX3 is a marker of osteogenic differentiation in mesenchymal stem cell. Suehiro et al. ${ }^{46}$ found that ZHX3 has an important role in the early stages of osteogenic differentiation. ZHX3 mRNA expression was upregulated after incubation with mesenchymal stem cell in the osteogenic induction medium, which was 3- to 5-fold higher compaerd with that in undifferentiated mesenchymal stem cell, ${ }^{46}$ but not upregulated during chondrogenic or adipogenic differentiation of mesenchymal stem cell.

The role of ZHX proteins in glomerular diseases was indicated in animal models ${ }^{40,47}$ and human kidney biopsies. ${ }^{40} \mathrm{ZHX}$ proteins are mostly located in non-nuclear regions in normal podocyte in heterodimer way and a minority of them are located in normal nuclear regions. Furthermore, ZHX3 protein was transiently downregulated before the onset of proteinuria. The recovery of ZHX3 expression was associated with migration of ZHX3 protein into the nucleus and the development of proteinuria in minimal change disease. ${ }^{40} \mathrm{ZHX} 3$ regulates the expression of podocyte gene directly or indirectly via other ZHX proteins. ${ }^{40}$ Sustainable downregulation of $\mathrm{ZHX} 3$ protein causes the reduction of WT1, Lmx1b and Pax2 genes. All these genes are crucial in focal glomerulopathy sclerosis. ${ }^{40,48}$

\section{FUTURE DIRECTIONS}

Current studies indicate that ZHX family members are crucial in the development and progression of human diseases. However, studies of ZHX family members are still premature and further studies are required. First of all, to further identify the role of ZHX family members in the development of diseases, genetically engineered mouse models should be generated. Transgenic, knockout and knockin ZHX mouse models are still not available. Second, it is still unclear about the signaling pathway of ZHX family. The downstream targets and upstream regulators of $\mathrm{ZHX}$ proteins are required to be further investigated. Finally, it's also unclear whether ZHX family members are therapeutic targets in $\mathrm{HCC}$ and other kinds of diseases. Some preclinical experiments are required to be performed to identify the potential ZHX-targeted therapies.

\section{CONFLICT OF INTEREST}

The authors declare no conflict of interest.

\section{ACKNOWLEDGMENTS}

This work was supported by the National Nature Science Foundation of China (81370662). 


\section{REFERENCES}

1 Barthelemy I, Carramolino L, Gutiérrez J, Barbero JL, Márquez G, Zaballos A et al. Zhx-1: a novel mouse homeodomain protein containing two zinc-fingers and five homeodomains. Biochem Biophys Res Commun 1996; 224: 870-876.

2 Kawata H, Yamada K, Shou Z, Mizutani T, Miyamoto K. The mouse zinc-fingers and homeoboxes (ZHX) family; ZHX2 forms a heterodimer with ZHX3. Gene 2003; 323: 133-140.

3 Kawata H, Yamada K, Shou Z, Mizutani T, Yazawa T, Yoshino M et al. Zinc-fingers and homeoboxes (ZHX) 2, a novel member of the ZHX family, functions as a transcriptional repressor. Biochem J 2003; 373: 747-757.

4 Yamada K, Kawata H, Shou Z, Hirano S, Mizutani T, Yazawa T et al. Analysis of zincfingers and homeoboxes (ZHX)-1-interacting proteins: molecular cloning and characterization of a member of the ZHX family, ZHX3. Biochem J 2003; 373: 167-178.

5 Yamada K, Printz RL, Osawa H, Granner DK. Human ZHX1: cloning, chromosomal location, and interaction with transcription factor NF-Y. Biochem Biophys Res Commun 1999; 261: 614-621.

6 Hirano S, Yamada K, Kawata H, Shou Z, Mizutani T, Yazawa T et al. Rat zinc-fingers and homeoboxes 1 (ZHX1), a nuclear factor-YA-interacting nuclear protein, forms a homodimer. Gene 2002; 290: 107-114.

7 Nagase T, Ishikawa K, Suyama M, Kikuno R, Hirosawa M, Miyajima N et al. Prediction of the coding sequences of unidentified human genes. XII. The complete sequences of 100 new cDNA clones from brain which code for large proteins in vitro. DNA Res 1998; 5: 355-364.

8 Munshi A, Dadheech S, Jain S, Joseph J, Al-Hazzani A, Alshatwi AA et al. Lack of association of G779A ZHX-2 gene variant with $\mathrm{HbF}$ levels in betathalassemia major. Eur J Haematol 2011; 86: 502-506.

9 Ovsyannikova IG, Kennedy RB, O'Byrne M, Jacobson RM, Pankratz VS, Poland GA et al. Genome-wide association study of antibody response to smallpox vaccine. Vaccine 2012; 30: 4182-4189.

10 Ishikawa K, Nagase T, Nakajima D, Seki N, Ohira M, Miyajima N et al. Prediction of the coding sequences of unidentified human genes. VIII. 78 new CDNA clones from brain which code for large proteins in vitro. DNA Res 1997; 4: 307-313.

11 Bird LE, Ren J, Nettleship JE, Folkers GE, Owens RJ, Stammers DK et al. Novel structural features in two $\mathrm{ZHX}$ homeodomains derived from a systematic study of single and multiple domains. BMC Struct Biol 2010; 10: 13.

12 Ogata-Kawata H, Yamada K, Uesaka-Yoshino M, Kagawa N, Miyamoto K. BS69, a corepressor interacting with $\mathrm{ZHX1}$, is a bifunctional transcription factor. Front Biosci 2007; 12: 1911-1926.

13 Yamada K, Osawa H, Granner DK. Identification of proteins that interact with NF-YA. FEBS Lett 1999; 460: 41-45.

14 Yamada K, Kawata H, Matsuura K, Shou Z, Hirano S, Mizutani T et al. Functional analysis and the molecular dissection of zinc-fingers and homeoboxes 1 (ZHX1) Biochem Biophys Res Commun 2002; 297: 368-374.

15 Nagel S, Schneider B, Meyer C, Kaufmann M, Drexler HG, Macleod RA et al. Transcriptional deregulation of homeobox gene ZHX2 in Hodgkin lymphoma. Leukemia Res 2012; 36: 646-655.

16 Tomita T, Tanaka S, Morohashi Y, Iwatsubo T. Presenilin-dependent intramembrane cleavage of ephrin-B1. Mol Neurodegener 2006; 1: 2.

17 Gargalovic PS, Erbilgin A, Kohannim O, Pagnon J, Wang X, Castellani L et al. Quantitative trait locus mapping and identification of Zhx2 as a novel regulator of plasma lipid metabolism. Circ Cardiovasc Genet 2010; 3: 60-67.

18 Perincheri S, Dingle RW, Peterson ML, Spear BT. Hereditary persistence of alphafetoprotein and $\mathrm{H} 19$ expression in liver of $\mathrm{BALB} / \mathrm{CJ}$ mice is due to a retrovirus insertion in the Zhx2 gene. Proc Natl Acad Sci USA 2005; 102: 396-401.

19 Yamada K, Ogata-Kawata H, Matsuura K, Kagawa N, Takagi K, Asano K et al. ZHX2 and $\mathrm{ZHX3}$ repress cancer markers in normal hepatocytes. Front Biosci (Landmark Ed) 2009; 14: 3724-3732.

20 Belayew A, Tilghman SM. Genetic analysis of alpha-fetoprotein synthesis in mice. Mol Cell Biol 1982; 2: 1427-1435.

21 Yue X, Zhang Z, Liang X, Gao L, Zhang X, Zhao D et al. Zinc fingers and homeoboxes 2 inhibits hepatocellular carcinoma cell proliferation and represses expression of Cyclins A and E. Gastroenterology 2012; 142: 1559-70 e2.

22 Shen H, Luan F, Liu H, Gao L, Liang X, Zhang L et al. ZHX2 is a repressor of alphafetoprotein expression in human hepatoma cell lines. J Cell Mol Med 2008; 12: 2772-2780.

23 Hu SW, Zhang M, Xue L, Wen JM. Regulation effect of zinc fingers and homeoboxes 2 on alpha-fetoprotein in human hepatocellular carcinoma. Gastroenterol Res Pract 2013; 2013: 101083.

24 Morford LA, Davis C, Jin L, Dobierzewska A, Peterson ML, Spear BT et al. The oncofetal gene glypican 3 is regulated in the postnatal liver by zinc fingers and homeoboxes 2 and in the regenerating liver by alpha-fetoprotein regulator 2 . Hepatology 2007; 46: 1541-1547.

25 Weng M-Z, Zhuang P-Y, Hei Z-Y, Lin P-Y, Chen Z-S, Liu Y-B et al. ZBTB20 is involved in liver regeneration after partial hepatectomy in mouse. Hepatobiliary Pancreat Dis Int 2014; 13: 48-54.

26 Lv Z, Zhang M, Bi J, Xu F, Hu S, Wen J et al. Promoter hypermethylation of a novel gene, ZHX2, in hepatocellular carcinoma. Am J Clin Pathol 2006; 125: 740-746.

27 Wang J, Liu D, Liang X, Gao L, Yue X, Yang Y et al. Construction of a recombinant eukaryotic human $Z H X 1$ gene expression plasmid and the role of ZHX1 in hepatocellular carcinoma. Mol Med Rep 2013; 8: 1531-1536.

$28 \mathrm{Hu} \mathrm{S}$, Zhang M, Lv Z, Bi J, Dong Y, Wen J et al. Expression of zinc-fingers and homeoboxes 2 in hepatocellular carcinogenesis: a tissue microarray and clinicopathological analysis. Neoplasma 2007; 54: 207-211.

29 Garner C, Mitchell J, Hatzis T, Reittie J, Farrall M, Thein SL et al. Haplotype mapping of a major quantitative-trait locus for fetal hemoglobin production, on chromosome 6q23. Am J Hum Genet 1998; 62: 1468-1474.

30 de Andrade TG, Peterson KR, Cunha AF, Moreira LS, Fattori A, Saad ST et al. Identification of novel candidate genes for globin regulation in erythroid cells containing large deletions of the human beta-globin gene cluster. Blood Cells Mol Dis 2006; 37: 82-90.

31 De Andrade T, Moreira L, Duarte A, Lanaro C, De Albuquerque D, Saad S et al. Expression of new red cell-related genes in erythroid differentiation. Biochem Genet 2010; 48: 164-171.

32 Harousseau JL, Shaughnessy J Jr., Richardson P. Multiple myeloma. Hematology Am Soc Hematol Educ Program 2004: 237-256.

33 Shaughnessy J Jr., Zhan F, Barlogie B, Stewart AK. Gene expression profiling and multiple myeloma. Best Pract Res Clin Haematol 2005; 18: 537-552.

34 Armellini A, Sarasquete ME, Garcia-Sanz R, Chillon MC, Balanzategui A, Alcoceba M et al. Low expression of ZHX2, but not RCBTB2 or RAN, is associated with poor outcome in multiple myeloma. Br J Haematol 2008; 141: 212-215.

35 Legartova S, Harnicarova-Horakova A, Bartova E, Hajek R, Pour L, Kozubek S et al. Expression of RAN, ZHX-2, and CHC1L genes in multiple myeloma patients and in myeloma cell lines treated with HDAC and Dnmts inhibitors. Neoplasma 2010; 57: 482-487.

36 Nagel S, Schneider B, Rosenwald A, Meyer C, Kaufmann M, Drexler HG et al. t( $4 ; 8)$ (q27;q24) in Hodgkin lymphoma cells targets phosphodiesterase PDE5A and homeobox gene ZHX2. Genes Chromosomes Cancer 2011; 50: 996-1009.

37 Hystad ME, Myklebust JH, oslash TH, Sivertsen EA, Rian E, Forfang L et al. Char acterization of early stages of human $B$ cell development by gene expression profiling. J Immunol 2007; 179: 3662-3671.

38 Nagel S, Meyer C, Kaufmann M, Drexler HG, MacLeod RA. Deregulated FOX genes in Hodgkin lymphoma. Genes Chromosomes Cancer 2014; 53: 917-933.

39 Moen CJ, Tholens AP, Voshol PJ, de Haan W, Havekes LM, Gargalovic P et al. The Hyplip2 locus causes hypertriglyceridemia by decreased clearance of triglycerides. J Lipid Res 2007; 48: 2182-2192.

40 Liu G, Clement LC, Kanwar YS, Avila-Casado C, Chugh SS. ZHX proteins regulate podocyte gene expression during the development of nephrotic syndrome. J Biol Chem 2006; 281: 39681-39692.

41 Johansen CT, Wang J, Mclntyre AD, Martins RA, Ban MR, Lanktree MB et al. Excess of rare variants in non-genome-wide association study candidate genes in patients with hypertriglyceridemia. Circ Cardiovasc Genet 2012; 5: 66-72.

42 Wu C, Qiu R, Wang J, Zhang H, Murai K, Lu Q et al. ZHX2 Interacts with Ephrin-B and regulates neural progenitor maintenance in the developing cerebral cortex. $J$ Neurosci 2009; 29: 7404-7412.

43 Georgakopoulos A, Litterst C, Ghersi E, Baki L, Xu C, Serban G et al. Metalloproteinase/Presenilin1 processing of ephrinB regulates EphB-induced Src phosphorylation and signaling. EMBO J 2006; 25: 1242-1252.

44 Qiu R, Wang X, Davy A, Wu C, Murai K, Zhang H et al. Regulation of neural progenitor cell state by ephrin-B. J Cell Biol 2008; 181: 973-983.

45 Fekete R, Bainbridge M, Baizabal-Carvallo JF, Rivera A, Miller B, Du P et al. Exome sequencing in familial corticobasal degeneration. Parkinsonism Relat Disord 2013; 19: $1049-1052$.

46 Suehiro F, Nishimura M, Kawamoto T, Kanawa M, Yoshizawa Y, Murata H et al. Impact of zinc fingers and homeoboxes 3 on the regulation of mesenchymal stem cell osteogenic differentiation. Stem Cells Dev 2011; 20: 1539-1547.

47 Clement LC, Liu G, Perez-Torres I, Kanwar YS, Avila-Casado C, Chugh SS et al. Early changes in gene expression that influence the course of primary glomerular disease. Kidney Int 2007; 72: 337-347.

48 Chugh SS. Transcriptional regulation of podocyte disease. Transl Res 2007; 149: 237-242. 